\title{
A Cognitively-Inspired Model for Making Sense of Hasse Diagrams
}

\author{
Dimitra BOUROU $^{\mathrm{a}, \mathrm{b}, 1}$ Marco SCHORLEMMER $^{\mathrm{a}, \mathrm{b}}$ and Enric PLAZA ${ }^{\mathrm{a}}$ \\ ${ }^{a}$ Artificial Intelligence Research Institute, IIIA-CSIC, Bellaterra, Catalonia, Spain \\ ${ }^{\mathrm{b}}$ Dept. Ciències de la Computació, Universitat Autònoma de Barcelona, \\ Bellaterra, Catalonia, Spain
}

\begin{abstract}
.
In this paper, we present a model of the sense-making process for diagrams, and describe it for the case of Hasse diagrams. Sense-making is modeled as the construction of networks of conceptual blends among image schemas and the diagram's geometric configuration. As a case study, we specify four image schemas and the geometric configuration of a Hasse diagram, with typed FOL theories. In addition, for the diagram geometry, we utilise Qualitative Spatial Reasoning formalisms. Using an algebraic specification language, we can compute conceptual blends as category-theoretic colimits. Our model approaches sense-making as a process where the image schemas and the diagram geometry both structure each other through a complex network of conceptual blends. This yields a final blend in which the sort of inferences we confer to diagrammatic representations emerge. We argue that this approach to sense-making in diagrams is more cognitively apt than the mainstream view of a diagram being a syntactic representation of some underlying logical semantics. Moreover, our model could be applied to various types of stimuli and is thus valuable for the general field of AI.
\end{abstract}

Keywords. diagrammatic reasoning, conceptual blending, conceptual meaning, image schema, first-order logic, formal specification, sense-making.

\section{Introduction}

Sense-making refers to the process by which we structure our percepts into constructs that are more meaningful for us. In this work, we model the sense-making of diagrams as conceptual blends of their geometric configurations, with image schemas. The latter reflect early embodied sensorimotor experiences [1,2]. To the best of our knowledge, modeling the sense-making of diagrams in this manner is a novel contribution, which could be of value for fields pertaining to human-human or human-machine communication by means of graphical aids.

To illustrate our approach, take for instance the Hasse diagram of Fig. 1 (left). Its geometric configuration comprises points and lines, with each line intersecting with one pair of points. We propose that some of the possible ways one could make sense of this diagram are that points $h, e, b$ and $a$ form a path with direction from $h$ to $a$, or that $h$ with

\footnotetext{
${ }^{1}$ Corresponding Author: Dimitra Bourou, Artificial Intelligence Research Institute (IIIA-CSIC), Campus UAB, Carrer de Can Planes, Zona 2, 08193 Bellaterra, Barcelona, Spain; e-mail: dbourou@iiia.csic.es
} 


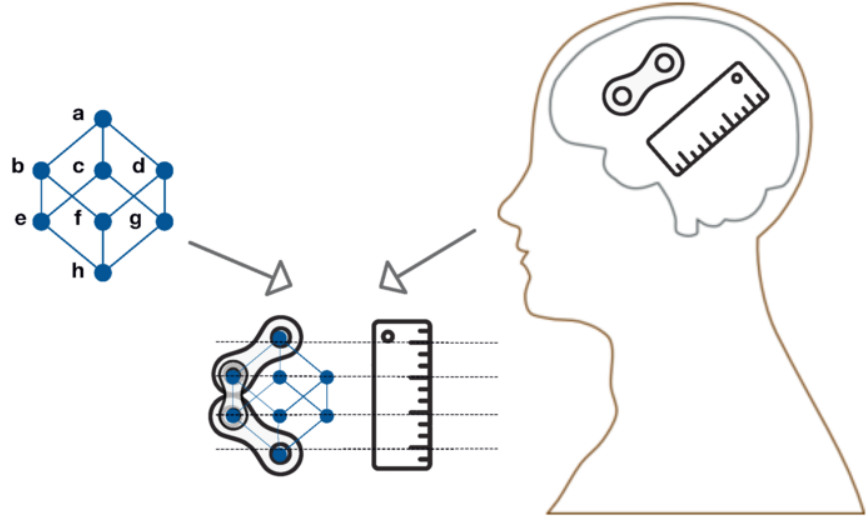

Figure 1. Conceptual blend of a Hasse diagram. The LINK or SCALE image schemas (right) are blended with the geometric configuration (left), yielding the Hasse diagram as we make sense of it (bottom).

$e, e$ with $b$, and $b$ with $a$, form pairs of entities symmetrically linked by lines. The diagram may also be understood as a scale of four grades, each grade consisting of the points on the same horizontal axis, e.g., grade 2 would comprise points $e, f$ and $g$. Finally, some or all the above conceptualisations could be applied jointly. These conceptualisations allow the emergence of some inferences on the diagram, such as $h$ and $a$ being associated, as parts of the same path, or $d$ being transitively on a higher grade than $h$.

This variety of understandings of the diagram leads to different conclusions, depending on whether the 'path', 'link', or 'scale' conceptualisation, or all of them, are at play; each imbues a different sense on the diagram, i.e., direction, symmetric association, and quantity, respectively. This shows that diagrams, taken as geometric configurations, do not bring up a unique way of making sense of them. We believe these alternative senses can be modeled as a result of combining different image schemas with each other and with the geometric configuration. The aforementioned concepts correspond directly with their homonymous image schemas and can thus be formalised as such. Appropriate correspondences between the two schemas, and of the schemas with the geometric configuration, are responsible for constraining the exact form of the image schemas, or their blends, that will contribute to structuring the geometry. For example, the instance of SCALE corresponding to the Hasse diagram of Fig. 1 would have four grades. Subsequently, given these correspondences, the final blend between image schemas and geometry can be constructed, and inferences can arise thereby.

Our proposal is to model the aforementioned sense-making process as follows: A configuration consists only of geometric entities. The ability to do inference with the diagram is not a result merely of its geometry; the sense-making, which makes inference possible, arises when image schemas, with their internal structure, are blended with each other, and with the geometry, structuring it into a meaningful diagram (Fig. 1). Here, we describe our model of sense-making for the case of Hasse diagrams. We believe that viewing sense-making as emerging from an entire network of interrelated concepts paves the way towards an efficient and cognitively plausible model of conceptual meaning. Such a model would comprise only a few image schemas and blending principles, but would be able to give rise to a host of different instantiations of image schemas and various interpretations for a given stimulus. 


\section{Background}

The literature of diagrammatic reasoning has been valuable for formally studying the informational content and the efficacy of diagrams for inference. In order to reach such conclusions, a one-to-one correspondence between the geometry (syntax) and the semantics of the diagram is typically assumed. However, as explained above, a certain geometric configuration does not always evoke a unique understanding. Furthermore, the interpretation of diagrams entails a constructive and imaginative process [3]. This is in line with the process of sense-making, which we model computationally in this work.

Sense-making is defined in the literature of enactive cognition as the process of an autonomous agent bringing its own original meaning upon its environment [4]. Image schemas are fundamental for such a process, because they have the capacity to organise and structure our experience [2, p. 372]. Image schemas are mental structures, formed early in life, that constitute structural contours of repeated sensorimotor contingencies such as SUPPORT, VERTICALITY, and BALANCE. For example, the meaning of balance emerges through the repeated embodied experience of different kinds of balance, which leads to the formation of a mental structure reflecting what is invariant among them [1, p. 74-75]. Image schemas are gestalts; they consist of components in a specific relational structure, which can be projected onto another domain and guide inference in it. This is related to the phenomenon of mental visualization, i.e., seeing in our "mind's eye" a generic chair when reading the word 'chair' [5,6]. Mental visualization is necessary for inference and prediction, and image schemas have been proposed to enable such visualization [7, pp. 513, 519-520].

One way to approach the aforementioned projection of image schema structure onto other domains is through conceptual blending. Conceptual blending is a process by which several mental spaces - coherent and integrated chunks of information that underlie cognition and which comprise entities, and relations or properties that characterise themare put into correspondence with each other via cross-space relations, so as to be integrated into a blend with novel structure [8]. The original mental spaces can be referred to as input spaces, and the resulting blend as blended space.

In Fig. 1, the input spaces involved are the Hasse geometric configuration and the LINK and SCALE schemas. The blend integrates both image schemas with the geometrric configuration, capturing one way we can make sense of the diagram, i.e., as comprising a set of symmetrically associated entities (owing to LINK), and, at the same time, a single graded directional structure (owing to SCALE).

\section{Approach}

In this paper, we model the sense-making of a sensory stimulus, i.e., a diagram, by means of cross-space correspondences between an input space reflecting this stimulus, and input spaces reflecting instances of image schemas. These correspondences guide the construction of blended spaces, integrating image schemas with some substructure of the stimulus. The emergent, integrated structure of this blended space enables novel inferences that were not possible in each input space alone. The entirety of the complex network of correspondences between spaces, and the blends emerging, reflect the sense-making of the given stimulus (here, geometric configuration). 
To model this network of blends, we provide: (a) A formal specification of the geometry of the diagram. Qualitative Spatial Reasoning (QSR) formalisms model several aspects of spatial configurations at a level compatible with human perception. Here, we use QSR and typed first-order logic (FOL) theories to capture the geometry of the diagram. (b) Formal specifications of the structure of the relevant image schemas, also by means of typed FOL theories. (c) A formalisation of suitable correspondences between image-schema structures and the geometric configuration. Given such correspondences, each blend can be modeled as a particular kind of category-theoretical colimit [9].

\subsection{Diagrammatic notation and geometric configuration}

A Hasse diagram represents a partially ordered set (poset). It consists of edges and vertices, drawn as points and lines. Each point represents one element of the poset. Assuming elements $x, y$ and $z$ of the poset, ordered by the ' $<$ ' relation, then the syntactic rules are that if $x<y$, then $x$ is shown in a lower position than $y$ in the diagram, and that $x$ and $y$ are connected by a line in the diagram iff: $x<y$ or $y<x$, and there is no element $z$ such that $x<z$ and $z<y$.

In order to describe this geometric configuration, we draw from formal systems of the QSR literature. Existing formalisms enable us to characterise spatial entities as points, lines, and regions, to describe their topological relations [10], and their qualitative position with respect to each other [11]. Concretely, the Hasse configuration of Fig. 1 has eight points ( $a$ to $h$ ) and twelve lines $(b a, e b$, etc.). Each line intersects with a pair of points. Below is a fragment of the specification of this configuration, which states some of the topological and orientation relations of its geometric entities. ${ }^{2}$

$\begin{array}{ll}\text { intersects }(b a, a) & \text { intersects }(b a, b) \\ \text { intersects }(e b, b) & \text { intersects }(e b, e) \\ \text { intersects }(h e, e) & \text { intersects }(h e, h)\end{array}$

$$
\begin{array}{r}
\text { right_front }(a, b) \\
\text { front }(b, e) \\
\text { left_front }(e, h)
\end{array}
$$

\subsection{Image schema formalisation}

To capture the structure of image schemas, we formalise their components and logical properties as typed FOL theories. In this effort, we were guided by conceptual descriptions of image schemas (mainly [1,2]), or experimental work, when available. For lack of space, we present here only the LINK and the VERTICALITY schemas in formal detail, and the remaining schemas in brief. ${ }^{3}$

\footnotetext{
${ }^{2}$ Predicates such as intersects state the topological relations as defined in [10], while predicates such as right_front state the orientation of entities as defined in [11]. Constants $a$ to $h$ are of type Point; constants $b a$, $e b$, etc. are of type Line. We note that the formalism proposed by Hernandez [11] offers various possible reference frames for orientation. These include frames depending on object functionalities, observers' viewpoint, or global, absolute frames like north, west etc. The latter is the approach we have followed, approaching all diagrams as they are typically presented on paper, with the upper part being front. Moreover, all QSR formalisms allow spatial reasoning. However, our goal here is to explore the reasoning afforded by blending the geometry with image schemas, and thus use the QSR system only for representation purposes.

${ }^{3}$ The complete formalisation of the blends modeling the sense-making of the Hasse diagram, and more kinds of diagrams, can be downloaded from https://drive.google.com/drive/folders/ 1jcQdJTOqbnAua3uXIgTEW8zV3kF_2R14?usp=sharing.
} 
LINK. The LINK schema pertains to the notion of association, either physical or abstract. The prototypical LINK schema associates two distinct, usually contiguous, entities with each other through a link. The relevant axioms are presented and explained below. ${ }^{4}$

$$
\begin{aligned}
& \forall s \in \text { LinkSchema }: \operatorname{linked}(\text { anEnt }(s), \text { anotherEnt }(s)) \\
& \forall x, y \in \text { Entity; } s \in \operatorname{LinkSchema}: \operatorname{linked}(x, y) \Leftrightarrow \\
& \quad(\operatorname{anEnt}(s)=x \wedge \text { anotherEnt }(s)=y) \vee(\operatorname{anEnt}(s)=y \wedge \operatorname{anotherEnt}(s)=x) \\
& \forall l \in \operatorname{Link} \exists ! s \in \operatorname{LinkSchema}: l=\operatorname{link}(s) \\
& \forall x, y \in \operatorname{Entity}: \operatorname{linked}(x, y) \Leftrightarrow \operatorname{linked}(y, x) \\
& \forall x \in \operatorname{Entity}: \neg \operatorname{linked}(x, x)
\end{aligned}
$$

PATH. The PATH schema is related to directionality and motion from a source towards a goal. It comprises a non-branching series of adjacent locations which connect the source with the goal. By the structure of the schema, it is obvious that, if someone is on a certain location of the path, then they have already traversed all prior locations. Therefore, the PATH schema is axiomatised as a total order; a collection of serially neighboring locations with the source and goal as endpoints.

VERTICALITY. The VERTICALITY schema obtains its structure from our experience of standing upright with our bodies resisting to gravity, or from perceiving upright objects like trees. The VERTICALITY schema reflects the axis of an upright object, so it must have a base as the bottom of the object [12]. Moreover, the axis may be merely mentally visualized by the observer; for example, when observing the sun, the horizon is the base, and a visualized vertical axis runs upward from the horizon, reaching the sun. Consequently, VERTICALITY is modeled simply as a unique vertical axis with its base. The base is a mark on the axis, such that no other mark on the axis can be placed above it.

$$
\begin{aligned}
& \forall s \in \operatorname{VerticalitySchema}: \operatorname{inAxis}(\text { base }(s), \operatorname{axis}(s)) \\
& \forall m \in \operatorname{Mark}: \neg \operatorname{above}(m, m) \\
& \forall s \in \operatorname{VerticalitySchema} ; m \in \operatorname{Mark}: \operatorname{inAxis}(m, \operatorname{axis}(s)) \wedge(m \neq \operatorname{base}(s)) \Rightarrow \operatorname{above}(m, \operatorname{base}(s))
\end{aligned}
$$

SCALE. The SCALE schema relates to a gradient of quantity. We believe that it comprises an ordered set of several grades, but, unlike VERTICALITY, it should not imply a particular geometric orientation. The SCALE schema has a directionality, and a cumulative property; if one has 15 euros, they also have 10 . This structure is modeled as a total order on grades.

\subsection{A formal model of sense-making}

Given the aforementioned formalisation of the input spaces involved, the sense-making of the Hasse configuration is modeled as the complex conceptual blending of several simpler blends.

\footnotetext{
${ }^{4}$ Elements of type LinkSchema are constituted of two components of type Entity and one component of type Link, which are obtained with functions anEnt, anotherEnt, and link, respectively (not shown). The axioms above state that that the two entities of a LinkSchema are always linked; that linked entities are always part of some LinkSchema; that a link is always part of a unique LinkSchema; and that the linked predicate is irreflexive and symmetric.
} 
One part of the network of blends at hand includes the input spaces of the PATH and the LINK schemas (Fig. 2). To blend these two schemas with each other, and with the geometric configuration, each pair of linked entities, and each pair of contiguous locations in a path, are put into correspondence with two points of the geometric configuration which intersect with the same line. By extension, a sequence of points connected by lines in the Hasse configuration (e.g., points $h, e, b$, and $a$ in Fig. 1; left), can be put into correspondence with a particular instance of PATH by relating connected points (such as $h$ and $e, b$ and $e$, and $b$ and $a$ ) with contiguous locations of PATH, and end points (such as $h$ and a) with the source and the goal of PATH respectively. More precisely, these cross-space correspondences between the input spaces of the Hasse geometric configuration and the LINK or the PATH schema can be expressed as pairs of a binary relation $R$ between entities of the two spaces. For example, the points $h, e, b$, and $a$ are related through $R$ with the source, $l_{2}, l_{3}$, and goal locations of an instance of PATH as follows:

$$
R(\text { source }, h) \quad R\left(l_{2}, e\right) \quad R\left(l_{3}, b\right) \quad R(\text { goal }, a)
$$

All the aforementioned correspondences allow the construction of a network of blends (Fig. 2) and ultimately result in the integration of LINK, PATH, and the geometric configuration, into a final blend comprising an integrated geometric and image-schematic structure with both directed paths of consecutive points, as well as pairs of linked points. Notice that the structure of the geometric configuration contributes to shaping the precise form of the image schema instances, and how they are blended with each other. At the same time, as all input spaces (image schemas and geometry) are involved in an intricate network of correspondences and blends (Fig. 2), they all structure each other, resulting in a final blend that comprises an integration of structure from all input spaces.

In a similar way, the VERTICALITY and SCALE schemas also structure the Hasse diagram. The marks of an instance of VERTICALITY, and the grades of an instance of SCALE schema, are put into correspondence with points of the Hasse diagram that are in the same horizontal axis. Point $h$, which is geometrically lowest, corresponds to the base of VERTICALITY. Marks, or grades whereby one is ordered immediately above the other, correspond to pairs of points whereby one is oriented immediately 'front' (or 'left_front', or 'right_front') of the other. This blend comprises a single 'vertical scale', with four integrated marks-grades (henceforth called 'levels'), the lowest of which is the base. Points belong to these levels and can thus be oriented with respect to the down-up axis. The configuration is thus now imbued with both the sense of verticality, as well as the sense of quantity, from the VERTICALITY and SCALE schemas respectively.

In summary, the Hasse diagram, as we make sense of it, emerges from a complex conceptual network with the four aforementioned image schemas, and the Hasse geometry, as input spaces. The correspondences between various instances of these schemas and substructures of the Hasse configuration, yield the Hasse diagram as comprising several paths of linked points, arranged at several levels of generality along an upward vertical axis. Some of these elements of different paths are on the same level of generality. Moreover, there is a unique source ordered before all other elements of the diagram, and a unique goal ordered after all other elements. Mathematically, these blends are computed as category-theoretic colimits of typed FOL theories [9]. 


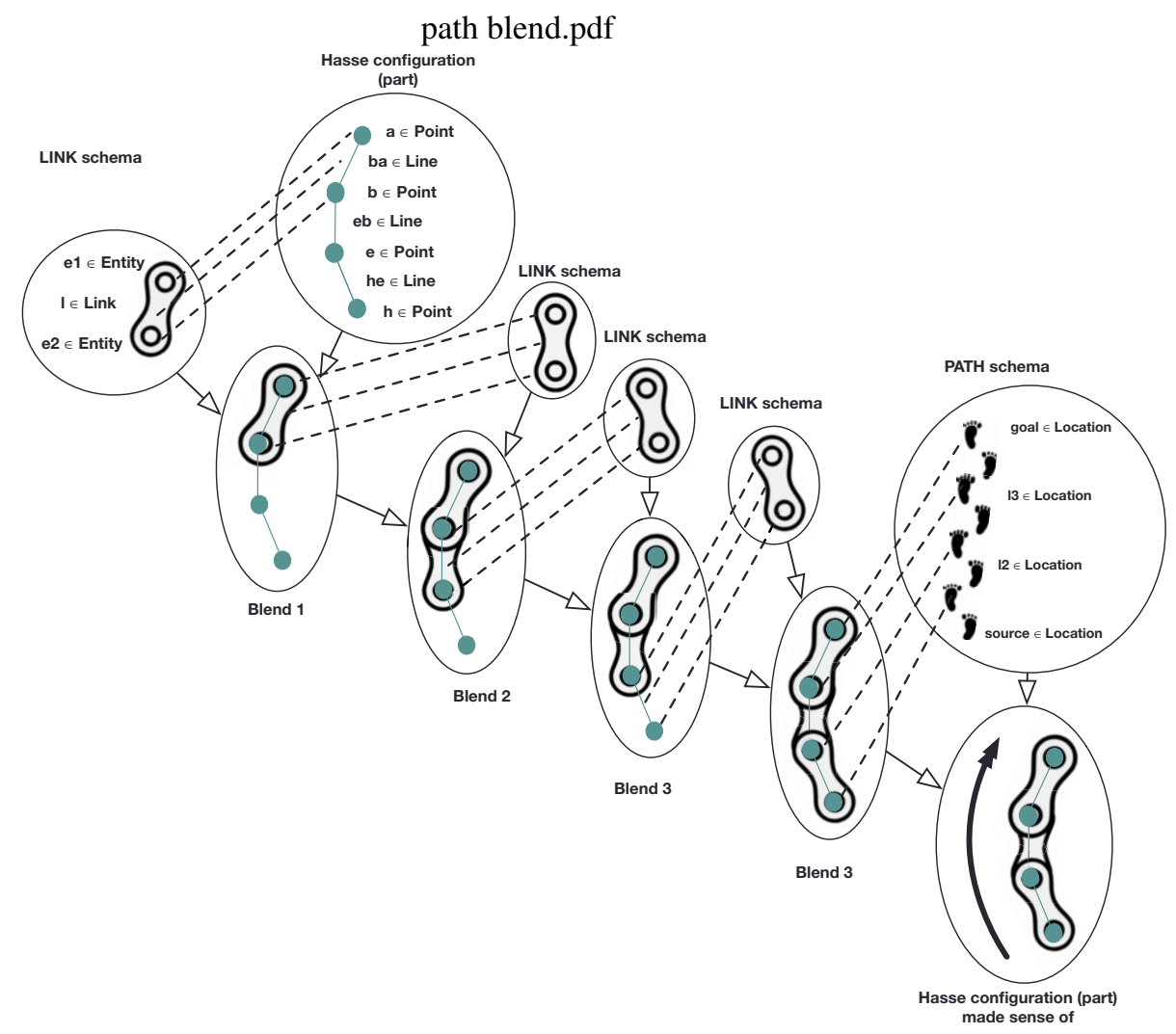

Figure 2. The network of blends that integrates the LINK and PATH schemas with part of the Hasse configuration. Correspondences between elements are shown with dashed lines.

\section{Related work}

In diagrammatic reasoning it is posited that the efficacy of diagrams lies in their sharing structural properties with their referents. These properties allow the observers to make inferences with these diagrams [13]. Therefore, the more the properties of the geometry of a diagram match the properties of its semantics, the more efficacious this diagram would be to represent this semantics, and to lead to valid inferences. Here, we expanded in this direction by modeling the origin of these properties as the blending of image schemas with the geometry of a diagram.

A few research groups have worked on formalising image schemas and the relations among them. Rodriguez and Egenhofer [14] provide a relational algebra inspired by the CONTAINER and SURFACE schemas, used to model, and reason about, spatial relations of objects in an indoor scene. Image schemas have also been used to model planning and actions of agents [15]. In the latter work, some image schemas were recursively defined in terms of other schemas. In both these works, the formalisations are merely inspired by image schemas, rather than faithful representations of their descriptions in the literature. Kuhn [16] formalised image schemas, and their combinations, as ontology relations using functional programming, in a relatively abstract manner. In a recent, comprehensive work, Hedblom [17] modeled image schemas as families of interrelated logical theories, 
with each schema comprising a combination of primitive components. QSR formalisms that capture the spatiotemporal content of schemas were used. In the present approach, we chose not to use such formalisms to capture the internal structure of image schemas.

Image schemas and blending have been used jointly mostly to model creative processes. Schorlemmer et al. [18] modeled the creative problem-solving process of tackling a riddle by way of a category-theoretic characterisation of blending, based on typed FOL theories of image schemas. In this work, image schemas were used to establish shared structure between different input spaces. In contrast, in a conceptual work by Falomir et al. [19], image schemas are used as input spaces, together with a QSR description of an icon, in order to blend them to interpret the latter. Importantly, here the stimulus mediates and structures the blending of image schemas with each other, as in our work. Finally, Embodied Construction Grammar allows the formalisation [20] and implementation [21] of language understanding by mapping components of specific schemas (image schemas, and others) to phonemes. The last two works are analogous to our own, modeling the sense-making of diagrams; except the stimulus made sense of is an icon and a spoken sentence respectively, instead of a diagram.

\section{Discussion}

In this paper we have presented a formal framework of the sense-making of diagrams as observers mentally structuring the geometry of diagrams by unconsciously projecting preexisting mental structures - i.e., image schemas - onto it, giving rise to inferences. We described examples of such inferences and pointed out that they are not fully determined by the geometry of the diagram, but also by image schemas in the observer's mind, and the way they are put into correspondence with this geometry.

Applying our framework to a Hasse diagram, we observe that the following facts can all be inferred from the geometric configuration : (a) point $a$ is above point $h$ (b) points $h$, $e, b$ and $a$ form a path and (c) points $b, c$ and $d$ are on the same level. To make inference (a), for instance, an observer may mentally visualize a physical path of linked locations, starting at location $h$, extending towards higher locations $e$ and $b$, up to $a$, which lies above $h$ and the rest of the locations traversed in the path. This mental visualization facilitates making the inference that $h<a$ directly from the Hasse diagram. Mental visualization is necessary for inference, and image schemas are the mental structures that enable it [7, pp. 513, 519]. Subjects are indeed able to make a correct one-step transitive inference on a given vertically-oriented diagram, without physically manipulating it [22], so mental visualization could be involved. More generally, the diagrammatic inferences that are captured in our blend network are: the transitive ordering of points in terms of their level, the inference that the source and the goal point of the PATH schema are ordered before and after all other points, respectively (corresponding semantically to the minimal and maximal element), and the existence of distinct instances of PATH schema (including all maximal chains).

Hasse diagrams indeed prioritise visualizing the structure of the order they represent, through a vertical organization, and explicit visualization of levels ${ }^{5}$ [23]. Following the assumption that the efficacy of diagrams results from shared properties between

\footnotetext{
${ }^{5}$ A Hasse diagram explicitly shows levels when elements with the same rank in the poset, i.e., same number of steps away from the minimum element, are placed in the same horizontal axis.
} 
their geometry and semantics [13], a Hasse diagram would be efficacious to represent a poset, because both its geometric configuration of shapes along a vertical axis, as well as its partial order semantics, share properties of transitivity and asymmetry. In our view, these properties may become cognitively salient by integrating -i.e., building crossspace correspondences between - the VERTICALITY and SCALE schemas with both the Hasse configuration and the poset semantics. In fact, it has been proposed that our understanding of abstract set theoretical notions also rests on image schemas [24]. The aforementioned inferences (transitivity, existence of maximal elements, minimal elements, and maximal chains) can be made through a process of integrating image schemas with the geometry and with the semantics of a Hasse diagram. This view is further supported by experiments showing that Hasse diagrams that are not upright, or do not show levels, take longer to be interpreted by subjects [25]. The precise role of the diagram semantics in the blend networks representing sense-making will be explored in future work.

The novelty of our framework lies in the fact that it is not merely conceptual but also written in a formal, computer-processable language. We contribute to the literature with a reusable set of formalized image schemas. Importantly, our framework is general enough to apply to any type of stimuli that is expressible in typed FOL. Furthermore, the entire framework could eventually be generalized in a representation-independent manner as described in [9]. Finally, by formalising the optimality principles — proposed to guide the construction of networks of blends that are desirable, i.e., lead to emergent structure that is useful for inference- we could obtain possible cross-space correspondences automatically, as in [26]. Such a framework would comprise an efficient and cognitively plausible model of sense-making, which we consider a valuable contribution to AI.

As for diagrams, the issue of generating and evaluating alternative blends for a given configuration, including those that model erroneous interpretations, i.e., inconsistent ones with the intended semantics, will be explored in the future. Such work would allow us to pin down what makes one diagram likely to be more accurately interpreted than another. This information could be of value for human-computer interaction because it could provide guidelines for the design of efficacious diagrammatic and graphical visualizations. For example, if a designer wants to visually represent some ordinal values, a tool based on our framework might recommend the use of a vertical geometric configuration and not a horizontal one. This is because a VERTICAL-SCALE is likely to map to such a configuration and lead to a blend with the intended semantics. Various such recommendations can be made precise thanks to our model and could contribute to new tools directed at designers.

\section{Acknowledgments}

The present research was supported by CORPORIS (PID2019-109677RB-I00) funded by Spain's Agencia Estatal de Investigación; by DIAGRAFIS (202050E243) funded by CSIC; and by the Ajuts a grups de recerca consolidats (2017 SGR 172) funded by AGAUR / Generalitat de Catalunya. 


\section{References}

[1] Johnson M. The Body in the Mind: The Bodily Basis of Meaning, Imagination, and Reason. University of Chicago Press; 1987.

[2] Lakoff G. Women, Fire, and Dangerous Things. University of Chicago Press; 1987.

[3] May M. Diagrammatic Reasoning and Levels of Schematization. In: Iconicity. A Fundamental Problem in Semiotics. NSU Press, Copenhagen; 1999. p. 175-194.

[4] Varela FJ. Organism: A Meshwork of Selfless Selves. In: Organism and the Origins of Self. Springer; 1991. p. 79-107.

[5] Jackendoff R. Semantics and Cognition. vol. 8. MIT Press; 1983.

[6] Jackendoff R. Consciousness and the Computational mind. The MIT Press; 1987.

[7] Mandler JM, Cánovas CP. On defining image schemas. Lang Cogn. 2014;6(4):510-532.

[8] Fauconnier G, Turner M. The Way We Think. Basic Books; 2002.

[9] Schorlemmer M, Plaza E. A uniform model of computational conceptual blending. Cogn Syst Res. 2021;65:118-137.

[10] Egenhofer MJ, Herring JR. Categorizing binary topological relations between regions, lines, and points in geographic databases. Department of Surveying Engineering, University of Maine; 1991.

[11] Hernández D. Relative Representation of Spatial Knowledge: The 2-D Case. In: Cognitive and Linguistic Aspects of Geographic Space. Springer; 1991. p. 373-385.

[12] Serra Borneto C. Liegen and Stehen in German: A study in horizontality and verticality. In: Cognitive Linguistics in the Redwoods. Mouton de Gruyter; 1996. p. 459-506.

[13] Shimojima A. On the efficacy of representation [PhD dissertation]. Indiana University; 1996.

[14] Rodríguez MA, Egenhofer MJ. A comparison of inferences about containers and surfaces in small-scale and large-scale spaces. J Vis Lang Comput. 2000;11(6):639-662.

[15] St Amant R, Morrison CT, Chang YH, Mu W, Cohen PR, Beal C. An image schema language. North Carolina State University at Raleigh, Dept. of Computer Science; 2006.

[16] Kuhn W. An image-schematic account of spatial categories. In: Proc. International Conference on Spatial Information Theory. Springer; 2007. p. 152-168.

[17] Hedblom MM. Image schemas and concept invention: Cognitive, logical, and linguistic investigations. Springer; 2020.

[18] Schorlemmer M, Confalonieri R, Plaza E. The Yoneda Path to the Buddhist Monk Blend. In: Proc. of the Joint Ontology Workshops; 2016. .

[19] Falomir Z, Plaza E. Towards a model of creative understanding: Deconstructing and recreating conceptual blends using image schemas and Qualitative Spatial Descriptors. Ann Math Artif Intell. 2019;88:457-477.

[20] Bergen B, Chang N. Embodied construction grammar in simulation-based language understanding. In: Construction grammars: Cognitive grounding and theoretical extensions. vol. 3. John Benjamins; 2005. p. 147-190.

[21] Bryant JE. Best-Fit Constructional Analysis [PhD dissertation]. EECS Department, University of California, Berkeley. Magdeburg, Germany; 2008. http://www2. eecs.berkeley.edu/Pubs/ TechRpts/2008/EECS-2008-100.html.

[22] Shimojima A, Katagiri Y. An eye-tracking study of exploitations of spatial constraints in diagrammatic reasoning. Cogn Sci. 2013;37(2):211-254.

[23] Demey L, Smessaert H. The relationship between Aristotelian and Hasse diagrams. In: Proc. of the International Conference on Theory and Application of Diagrams. Springer; 2014. p. 213-227.

[24] Lakoff G, Núñez RE. Where mathematics comes from: How the embodied mind brings mathematics into being. AMC. 2000;10(12):720-733.

[25] Körner C, Albert D. Comprehension efficiency of graphically presented ordered sets. In: Current psychological research in Austria. Proc. of the 4th Scientific Conference of the Austrian Psychological Society. Graz: Akademische Druck - u. Verla; 2001. p. 179-182.

[26] Pereira FC, Cardoso A. Optimality principles for conceptual blending: A first computational approach. AISB J. 2003;1(4):351-369. 